María de los Angeles Bacigalupe*

\title{
Los estudios del comportamiento humano en la construcción de la neurociencia educacional
}

\section{Studies on human behavior in the construction of educational neuroscience}

\begin{abstract}
We discuss the building of educational neuroscience and its relationships with some studies on human behavior that serve the construction of the educational neuroscience but not define it. We understand that behavior is a relationship between the organism and the environment and that learning is a form of behavior. We put forward a transdisciplinar approach (not an applicationist approach which means to apply the knowledge to one discipline to another discipline) to work on the relationships between neurosciences and education and analyze the way the studies on human behavior could collaborate to build the educational neuroscience. We discuss problems such as the problem of generation of neuromyths from pathological cases of neurosciences and psychology. Knowing that every people have biology and that this is not minor information to the time of educating persons, we think that educational neuroscience has a broad field of knowledge to develop to discuss educational issues.
\end{abstract}

Keywords | educational neuroscience, neuromyth, learning, education, human behavior.

Resumen | En el presente artículo nos proponemos discutir la construcción de la neurociencia educacional y sus relaciones con algunos estudios del comportamiento humano que resultan importantes para su construcción pero no necesariamente para su definición. Entendemos el comportamiento como relación organismo-entorno y el aprendizaje como una forma de comportamiento. Proponemos una mirada transdisciplinaria y no aplicacionista de las relaciones entre neurociencias y educación, y analizamos la forma en que los estudios del comportamiento humano podrían colaborar en la construcción de la neurociencia educacional. Abordamos problemas tales como la generación de neuromitos a partir de casos patológicos de las neurociencias y la psicología. Sabiendo que todas las personas tenemos

Recibido: 11 de marzo de 2019.

Aceptado: 25 de mayo de 2020.

* Investigadora adjunta. Consejo Nacional de Investigaciones Científicas y Técnicas (CONICET, Argentina-División Etnografía Museo de la Plata), Facultad de Ciencias Naturales y Museo, UNLP.

Correo electrónico: mariabacigalupe@conicet.gov.mx

Bacigalupe, María de los Angeles. «Los estudios del comportamiento humano en la construcción de la neurociencia educacional.» Interdisciplina 8, $\mathrm{n}^{\circ} 22$ (septiembre-diciembre 2020): 223-245. DOI: https://doi.org/10.22201/ceiich.24485705e.2020.22.76427 
INTER DISCIPLINA

www.interdisciplina.unam. $\mathrm{mx}$
Volumen 8, número 22, (223-245), septiembre-diciembre 2020 DOI: https://doi.org/10.22201/ceiich.24485705e.2020.22.76427 María de los Angeles Bacigalupe

biología y que ese no es un dato menor a la hora de educar, consideramos que la neurociencia educacional tiene un amplio campo de conocimiento por desarrollar en la discusión constructiva con otras ciencias sociales para el abordaje de problemáticas educacionales.

Palabras clave | neurociencia educacional, neuromito, aprendizaje, educación, comportamiento humano.

\section{Introducción}

EN EL PRESENTE ARTÍCULO nos proponemos discutir la construcción de la neurociencia educacional y sus relaciones con algunos estudios del comportamiento humano que resultan importantes para su construcción pero no necesariamente para su definición.

La necesidad de plantear esta cuestión surge de la misma práctica educacional y de la necesidad de elaboración teórica de la cuestión ante la escasez de alternativas de respuesta en la bibliografía consultada.

Existen ciertos neuromitos que no son fáciles de erradicar o de transformar y uno de ellos es que estudiar algo que tenga que ver con la biología en educación está orientado necesariamente a la educación especial; este neuromito suele asociarse con otro en el cual se habla de plasticidad y aprendizaje en niños y adolescentes, pero se olvidan las responsabilidades de la educación en el ámbito de la adultez y de adultos mayores.

La neurociencia educacional es una transdisciplina que no tiene por qué confundirse con la educación especial, pero, habida cuenta de que algunos estudios comportamentales que contribuyen al conocimiento del aprendizaje y el cerebro se han desarrollado sobre el supuesto neuropsicológico no erróneo de que se conoce más de una función trabajando con personas donde esa función está afectada (supuesto actualmente prescindible ya que las técnicas actuales de exploración cerebral no requieren de la existencia de lesión para estudiar la función), muchas personas pueden terminar pensando que la neurociencia educacional es una rama de las neurociencias o de la educación que tiene su sentido de existencia solo cuando se trabaja en o sobre contextos de integración social, discapacidad y riesgo social por malnutrición y falta de estimulación, especialmente con niños. Es decir, en el saber popular suele asociarse el estudio de la biología en educación con la educación especial en niños.

Este pensamiento constituye un gran error que no respeta los principios iniciales de la neurociencia educacional, esto es, la posibilidad de plasticidad a lo largo y ancho de la curva normal (normal entendido estadísticamente) y el reconocimiento de que todas las personas, es más, todos los organismos, tenemos nuestra biología e interactuamos con un entorno biótico y abiótico con el que desarrollamos relaciones de co-construcción (Lewontin 2000; Meyer 1977). 
En este trabajo, entendemos el comportamiento como relación organismoentorno (Lahitte 1989; Piaget 1978) y a los individuos como organismos extendidos (Turner 2000) o sujetos extendidos (C. Garay, UNLP, comunicación personal).

El aprendizaje es un concepto clave dentro de estos procesos de extensión y comportamiento: de hecho, lo podemos entender como comportamiento de ciertos organismos, aquellos que poseen sistema nervioso y, por ende, que presentan la cualidad de plasticidad cerebral. Puede discutirse el aprendizaje en organismos sin sistema nervioso, pero eso queda por fuera de los objetivos de este trabajo. Nosotros entenderemos el aprendizaje como proceso cognitivocomportamental que ocurre en relación, lo cual involucra al menos dos subsistemas relacionados: el individuo y su entorno.

\section{Qué es la neurociencia educacional Neurociencia educacional}

En su edición extendida de la obra How People Learn de ya veinte años de antigüedad, Bransford, Brown y Cocking $(2000,4)$ señalan que la neurociencia: "is beginning to provide evidence for many principles of learning that have emerged from laboratory research, and it is showing how learning changes the physical structure of the brain and, with it, the functional organization of the brain".

Los autores mencionados opinan que la información y el conocimiento están creciendo de un modo nunca visto en la historia de la humanidad, lo cual implica que los objetivos de la educación cambian desde lo que en su momento fue valioso, es decir, conocer en el sentido de almacenar información disciplinar, a conocer en el sentido de obtener herramientas y desarrollar estrategias necesarias para adquirir conocimiento que permita a las personas pensar productivamente en los distintos ámbitos disciplinares y del saber. Esta perspectiva del aprendizaje no implica negar que los datos del conocimiento sean importantes para pensar y resolver problemas. Por el contrario, se sabe que la experticia ${ }^{1}$ se asocia con la posesión de un cúmulo importante de conocimiento en un área, pero ese cúmulo no es una colección de datos y hechos almacenados e inconexos sino que constituye un conocimiento usable, interconectado y organizado alrededor de conceptos centrales, asociado con ciertos contextos de aplicación y que puede ser base de comprensión y transferencia a otros contextos.

Coincidiendo con este cambio de perspectiva frente al conocimiento y el aprendizaje en los contextos actuales, De la Fuente Arias, Vera Martínez y Car-

1 N. del E.: Asociamos este término con el del inglés expertise: experiencia, pericia, habilidad, dominio. 
delle-Elawar (2012) señalan que la sociedad globalizada del conocimiento del siglo XXI supone cambios importantes en los modelos de trabajo e, incluso, de la vida cotidiana de las personas, que se vinculan con la revolución de las tecnologías de la información y la comunicación, trayendo consigo nuevas formas de comprender el conocimiento y las actividades humanas. Asimismo, RodríguezPonce (2009) considera que la sociedad del conocimiento, como resultante de la institucionalización y avance de la ciencia, se encuentra en continua construcción sobre la base de las tecnologías de la información y comunicación y se vuelve cada vez más competitiva en tanto el conocimiento es cada vez más universalmente accesible, provocando cambios en la vida humana y dinámica social como producto del inigualable desarrollo científico y tecnológico.

Esta situación trae aparejado un cambio necesario en la educación. Para Carreón Mendoza y Melgoza Ramos (2012) la educación se coloca como función clave en esta sociedad globalizada del conocimiento y la información, constituyendo la pieza del juego que articula los objetivos del desarrollo, los sistemas nacionales del conocimiento y los cambios de la institucionalidad universitaria.

El aprendizaje tiene un papel clave en este contexto. En su perspectiva que no ha perdido vigencia, Bransford et al. (2000) afirman que el aprendizaje mejora cuando: a) los docentes tienen en cuenta los conocimientos previos de los educandos, los usan como punto de partida y monitorean su cambio conceptual a lo largo del proceso de aprendizaje, y, cuando, b) los educadores ayudan a sus educandos a desarrollar una actitud activa de su propio proceso de aprendizaje vinculada con el desarrollo de autoconocimiento, autocontrol y metacognición. En este trabajo entendemos el concepto de metacognición como la habilidad de las personas para predecir su propia performance en determinadas tareas y automonitorear su nivel de experticia y maestría.

Desde la perspectiva de la autora de este artículo, esta aproximación metacognitiva del aprendizaje constituye una condición basada en el propio conocimiento de la propia motivación, que está en función del aprendizaje del trabajo en equipo, inter y transdisciplinario, y la resolución de problemas en grupos, competencias tan necesarias en el mundo actual del conocimiento.

La aproximación denominada brain based learning (aprendizaje basado en el cerebro, cfr. Caine y Caine 1994; Rodgers 2015) constituyó en su momento una idea innovadora y mantiene su relevancia actualmente dentro del campo del estudio del aprendizaje en el contexto educacional, intentando abordar una mirada metacognitiva de la enseñanza desde los conocimientos sobre el cerebro y la conducta. Caine y Caine (1994) proponen en su libro doce principios del aprendizaje basado en el cerebro, a saber: 1) el cerebro es un procesador en paralelo; 2) el aprendizaje involucra la fisiología completa de la persona; 3) la búsqueda del sentido de la experiencia es innata; 4) esa búsqueda ocurre a través de la or- 
ganización y categorización de la información en patrones; 5) las emociones son elementos clave en la formación de patrones; 6) el cerebro procesa la totalidad y las partes simultáneamente; 7) el aprendizaje involucra tanto la atención focalizada como la percepción periférica; 8) el aprendizaje involucra procesos conscientes e inconscientes; 9) habría al menos dos sistemas de memoria: un sistema espacial que no requiere necesariamente del ensayo para su adquisición, y otro sistema de aprendizaje de memoria (rote learning), dependiente de la repetición y más artificial; 10) se comprende y recuerda mejor cuando los hechos y las habilidades están integradas en la memoria espacial y la experiencia ordinaria; 11) el aprendizaje se estimula con el desafío y se inhibe con la amenaza y el miedo, $y, 12)$ cada cerebro es único. Tres elementos centrales de la experiencia de aprendizaje basada en el cerebro son los siguientes: a) inmersión orquestada en medioambientes complejos; b) alerta relajado (relaxed alertness), y, c) procesamiento activo (Rodgers 2015). La inmersión orquestada en ambientes complejos responde a la necesidad de planificar eventos experienciales y graduar la complejidad de esas experiencias para estimular el aprendizaje generando nuevas conexiones cerebrales y fortaleciendo otras; la orquestación es la disposición de oportunidades para que esto ocurra, y que suceda en condiciones de inmersión en el ambiente implica que el aprendiz está completamente rodeado, inmerso, en el tema de aprendizaje, lo cual, según Rodgers (2015), puede lograrse mediante experiencias de simulación y juego virtual. El alerta relajado implica que las experiencias de aprendizaje se planifican para que sean lo suficientemente desafiantes como para generar un nivel de estrés adecuado (eustress), evitando el estrés excesivo (distress) que es fisiológicamente perjudicial para la formación de memoria a largo plazo requerida en el aprendizaje. Finalmente, el procesamiento activo significa que el aprendizaje requiere no solo de la acción o experiencia sino de la reflexión sobre la experiencia, que es lo que permitirá conceptualizar la acción y construir el aprendizaje.

En una línea de estudio similar, Burgos (2015) destaca cinco principios que sostienen lo que se denomina mind brain education (MBE), un campo en crecimiento que vincula la investigación basada en evidencia de distintas disciplinas, incluyendo conocimiento de la biología, ciencias cognitivas y del desarrollo humano, con la educación, cuyo propósito es vincular la investigación con la práctica. Esos cinco principios pueden resumirse en las siguientes afirmaciones: a) cada cerebro es tan único como una huella digital; b) el contexto y las potencialidades individuales influyen en el aprendizaje; c) el cerebro tiene la capacidad de ser modificado por la experiencia; d) esta propiedad de neuroplasticidad ocurre a todo lo largo del ciclo vital y no solo en los grandes momentos del desarrollo como la niñez, y, e) se reconoce que el aprendizaje nuevo se construye sobre conocimiento previo que hace de andamiaje para las nuevas adquisiciones. 
El concepto de brain, mind and education science también es abordado por Tokuhama-Espinosa (2011), destacando que la ciencia así denominada es una nueva educación basada en el cerebro. La autora señala que si bien el campo de la MBE comenzó con un entrecruzamiento a través de la neurociencia cognitiva y la psicología del desarrollo, avanzó integrando la educación mediante la psicología educacional y la neurociencia educacional y hoy en día se constituye como un espacio transdisciplinario. Las disciplinas base de esta construcción transdisciplinaria, para la autora, son la neurociencia, la educación y la psicología.

Nuestros estudios sobre las relaciones entre neurociencias y educación datan del siglo pasado, y ya en una de nuestras publicaciones sobre el tema señalábamos que:

The relationship between neuroscience and education can provide important clues in relation to learning processes, and neuroscience can collaborate with pedagogy to provide some of the necessary tools for improving teaching methods. If the brain function helps to discover how people learn, then it may be possible for teaching methods to be improved. (Bacigalupe 2001/2, 12).

Lejos de sostener una mirada aplicacionista de las relaciones entre neurociencia y educación, sostenemos que la neurociencia educacional constituye un espacio transdisciplinario de trabajo donde no hay primacía de una disciplina sobre otra (neurociencia sobre educación) porque ya no se identifican como dos disciplinas sino como una transdisciplina (Pincham, Matejko, Obersteiner et al. 2014) que, por propia naturaleza, está en permanente construcción.

Entendemos por perspectiva transdisciplinaria una aproximación teórico-metodológica que pone énfasis en la apertura, flexibilidad y pluralismo disciplinares a fin de trascender los límites disciplinarios, con frecuencia enfocándose sobre problemas complejos del mundo real como centro y guía del proceso de investigación (Knox 2016).

Desde una aproximación más interdisciplinaria que transdisciplinaria, Butterworth y Tolmie (2014) entienden que son tres las disciplinas que constituyen el espacio de la neurociencia educacional: psicología, educación y neurociencias, lo cual concuerda con Tokuhama-Espinosa (2011) excepto en el aspecto clave, a nuestro parecer, de la mirada, que es interdisciplinaria en un caso (Butterworth y Tolmie 2014) y transdisciplinaria en el otro (Tokuhama-Espinosa 2011). La construcción de la neurociencia educacional ha seguido una historia donde en primer lugar hubieron intersecciones entre psicología y educación, y solo finalizando el siglo XX comenzaron a pensarse las posibilidades concretas de unir esta combinación inicial con las neurociencias (Butterworth y Tolmie 2014). Tres disciplinas 
están interactuando y por ende pueden aparecer problemas pues cada una tiene sus metodologías; sin embargo, Butterworth y Tolmie (2014) consideran que el diálogo entre disciplinas y la interoperabilidad (interoperability) de metodologías puede lograrse a través de la mediación de modelos computacionales de aprendizaje. A este respecto resulta interesante, y sorprendente a la vez, destacar que Tokuhama-Espinosa (2011) considera que la MBE supera a la neurociencia educacional y sería la forma de integrar y hacer transdisciplinaria su mirada.

Si bien consideramos valiosa la mirada interdisciplinaria de Butterworth y Tolmie (2014), apostamos por una concepción transdisciplinaria de la neurociencia educacional que no necesite apelar a una instancia integradora como la que propone Tokuhama-Espinosa (2011). Asimismo, no podemos dejar de destacar la importancia del aporte de Butterworth y Tolmie (2014) sobre la mediación de modelos computacionales del proceso de aprendizaje en esta construcción de la neurociencia educacional (Cfr., por ejemplo, Garay, Bacigalupe, Istvan et al. 2015).

Pincham et al. (2014) proponen que el espacio transdisciplinario de la neurociencia educacional no sea visto como una vía que une dos disciplinas distintas y que tiene una sola mano (de la neurociencia a la educación). Esta imagen pondría en evidencia la idea aplicacionista de la neurociencia, lo cual no es la intención con la construcción de la neurociencia educacional. Frente a esta imagen, los autores proponen la figura de una autopista de doble mano con una autonomía disciplinar. Sin embargo, diferimos con los autores sobre esta imagen y sobre todo cuando señalan cómo se llevaría a cabo. La imagen de una autopista de doble mano se asemeja a tender puentes entre dos disciplinas, mientras que nuestra propuesta es construir un nuevo espacio transdisciplinar. En segundo lugar, los autores indican que los pasos para llegar a construir la neurociencia educacional serían: a) identificar la necesidad educacional (investigadores y docentes); b) desarrollar una propuesta de investigación; c) probar en el contexto de clase, y, d) comunicar y evaluar junto a los docentes. Asimismo, concluyen esta idea afirmando: "At the heart of our approach is the notion that the educational neuroscientist not only engages in neuroscience research, but also assumes responsibility for translating that research or assessing its educational applicability" (Pincham et al. 2014, 29). Entendemos que esta idea vuelve al aplicacionismo y es por ello que disentimos con la propuesta aunque reconocemos sus avances respecto de otras.

Nuevamente nos encontramos con un intento de construcción que apela al aplicacionismo en Kalbfleisch (2015), quien define el objetivo de la neurociencia educacional de la siguiente manera:

After all, that is the goal of educational neuroscience - to apply the tools, metrics, and methods of neuroscience to questions and problems of human learning in order 
to inform aspects of curriculum design, pedagogy, and human performance in both formal and informal learning contexts. (Kalbfleisch 2015, 2).

Consideramos que constituye un problema para la construcción de la neurociencia educacional ver el objetivo de la misma no solo cuando se lo considera como aplicación, como en los casos anteriores, sino también cuando se habla de traducción. Butterworth y Tolmie (2014) señalan que el objetivo de la neurociencia educacional es lograr una investigación que puede o no entenderse como traductora (translational research) y la implementación de sus hallazgos para alcanzar una comprensión completa y profunda de los procesos de aprendizaje y su coordinación con las prácticas de enseñanza. En caso de que esa investigación de la que hablan los autores sea fruto de la propia neurociencia educacional (y no de las neurociencias como disciplinas separadas de la educación), sería muy beneficioso para los objetivos educacionales, aunque en la propuesta observamos el peligro de separar con esta idea de traducción lo que es teoría e investigación de lo que es la práctica educacional y, de este modo, repetir tradicionales disociaciones aunque ahora dentro de la misma neurociencia educacional. Sin despreciar esta postura, consideramos que un paso más favorecedor para la construcción de la neurociencia educacional sería generar su propio conocimiento de un modo transdisciplinario que, cualitativamente, es diferente a una interdisciplina, y que esta producción de conocimiento sea colaborativa entre docentes e investigadores: de hecho, dudamos de que un investigador educacional que no está en contacto con la realidad educativa pueda producir conocimiento útil para la educación.

De este modo, las interrelaciones entre neurociencias y educación no son tan simples de definir y, menos aún, de llevar a cabo. Cabe considerar pros and cons de esta interrelación (Varma, McCandliss y Schwartz 2008) para lograr una salida fructífera a los problemas de la educación.

Los problemas abordados son de la educación pero eso no implica que haya una dominancia de la educación sobre la neurociencia, pues sin la mirada que aporta el conocimiento neurocientífico muchos de estos problemas quizás ni siquiera podrían verse.

Lo que se sugiere es el logro de un diálogo co-construido entre neurociencias y educación, que requiere del planteamiento de un marco conceptual donde ambas disciplinas se vean beneficiadas (Dommett, Devonshire, Plateau et al. 2011). Así, Fischer y Heikkinen $(2010,251)$ señalan que: "mind, brain and education support the development of an educational neuroscience that are strongly interdisciplinary, contextualized, and pragmatic".

Claramente vemos en estos autores el objetivo de construir una neurociencia educacional que sea un espacio nuevo de producción de conocimiento útil, 
con una orientación pragmática, y no una aplicación de los conocimientos de las neurociencias a los contextos educacionales.

Es importante destacar que con orientación pragmática queremos referirnos a un punto de vista teórico-práctico y a la realización de la phronesis aristotélica (Cfr. Aristóteles 1970/ s. IV aC) y no a una perspectiva meramente práctica que entienda a los educadores como técnicos o aplicadores.

Revisar los propios presupuestos constituye una práctica clave en la constitución de la transdisciplina de la que estamos hablando. En este sentido, recuperamos la pregunta que Pincham et al. $(2014,28)$ formulan en su artículo: "What benefit, if any, can neuroscience add to understanding learning processes, improving educational practices and enhancing student outcomes?".

La necesidad de conocer el cerebro para la educación está claramente expresada por Spitzer $(2012,1)$ cuando dice que: "Neuroscience and education is like physics and architecture: Physics alone does not tell you how to built any structure in question, but you cannot build disregarding physical knowledge and principles". No obstante, diferimos con el autor cuando señala que entender el aprendizaje es entender el cerebro, pues sostenemos que tanto el aprendizaje (como el comportamiento) involucra relaciones entre el individuo y su entorno, como que el cuerpo del individuo está involucrado, recordando con esta última afirmación la hipótesis del marcador somático de Antonio Damasio (2008) y las teorías de Lawrence Barsalou (2010) sobre lo que denomina grounded cognition o cognición fundada.

Contrariamente a uno de los mitos creados con base en el entusiasmo y malentendimiento del significado de los estudios del cerebro, no somos un cerebro en una cubeta sino que nuestro cerebro depende de una acción corporizada (embodied) para desarrollarse (Fischer y Heikkinen 2010). Bien lo dice con una comparación Hruby $(2012,6)$ cuando critica el mal-entendimiento (tema que abordamos en la próxima sección de este artículo) cuando se habla del cerebro que aprende: "We are unlikely to tell a friend, 'My brain is going shopping', simply because we require our brain to do so".

No es el cerebro el que aprende sino que el aprendizaje, como comportamiento y como proceso, ocurre en la interacción entre el individuo y su entorno, entendiendo que el individuo no incluye solo su cerebro sino su cuerpo entero para aprender.

\section{Neuromitos}

Con la intención de poner el problema en su justo lugar, sin un optimismo exagerado ni un pesimismo devastador, y con una actitud que los autores llaman escepticismo saludable (healthy skepticism) y optimismo prudente (cautious optimism), Varma et al. (2008) diagraman un escenario de potenciales oportunidades 
en la interrelación neurociencias-educación a partir de ciertas áreas de preocupación que se agrupan en científicas y pragmáticas. Las preocupaciones científicas se refieren a la naturaleza diferencial de las ciencias de la educación y de las neurociencias en cuanto a métodos, datos, teoría y enfoque que hacen preguntarnos si esas diferencias pueden ser tan incompatibles que no permitan un diálogo. Las preocupaciones del nivel pragmático se refieren a los costos de investigación, la duda sobre si es el momento apropiado (si sabemos o no suficiente sobre neurociencias en este momento para ponernos a pensar en tender puentes con educación), quién tomará el control (¿qué pasa si las neurociencias toman el control sobre la educación?), y las consecuencias de esta intersección, ya que mucho de lo que se sabía sobre neurociencia en un momento devino en neuromito.

Se llama neuromito en educación a una concepción generada por una comprensión equivocada, una lectura erróneamente interpretada o una cita de alguien mal entendida acerca de hechos científicamente establecidos por la investigación neurocientífica, que tiene el objetivo de hacer a ese conocimiento aplicable en la educación y otros contextos (Dekker, Lee, Howard-Jones et al. 2012). Los neuromitos incluyen historias, relatos e ideas sobre el cerebro y las enfermedades relacionadas con el cerebro, algunos de los cuales están tan arraigados en las conversaciones cotidianas que gran parte de la población da por sentado que así es la realidad (Jarrett 2015).

En su artículo, Dekker et al. (2012) relatan una experiencia con docentes donde observaron que los neuromitos aparecían con gran incidencia en los docentes que mostraban un interés particularmente alto en las relaciones entre neurociencias y enseñanza, lo cual resulta realmente alarmante, debido a que estos docentes bienintencionados pueden intentar implementar en la práctica pedagógica del aula de clases ideas sobre el cerebro que son equivocadas. Asimismo, los autores reportan con preocupación que los docentes que quieren aprender sobre las relaciones entre investigaciones neurocientíficas y aplicaciones en el aula son propensos a buscar información en los medios de información populares.

Frente a la evidencia de la existencia de neuromitos que puede conducir a la educación a convertirse en cliente de la rápida comercialización de productos del mercado es que Dommett (2011) propone un diálogo co-construido entre ambas disciplinas. El autor relata su experiencia con un grupo de docentes enseñando conceptos de neurociencias y concluye que si bien algunos educadores manifestaron insatisfacción porque esperaban cierta estructura que los orientara en la práctica áulica, en general los resultados fueron satisfactorios para los destinatarios al señalar que habían adquirido conocimiento relevante. Si bien en esta experiencia hay un intento de dialogar y no de aplicar conocimientos de una disciplina (neurociencia) a la práctica educacional, puede observarse la in- 
certidumbre en el desarrollo efectivo de estas prácticas de transdisciplina tanto desde los destinatarios como desde los proponentes del estudio.

En su artículo, Dommett (2011) propone, por un lado, que no haya un liderazgo de una disciplina por sobre otra; por otro, que no haya una guía o consejo sobre la aplicación de resultados de neurociencia al aula de clases.

En teoría estos dos postulados son ideales pero en la práctica consideramos que solo se pueden lograr con la confirmación de un espacio nuevo, transdisciplinario, como hemos señalado más arriba.

Una cuestión importante a destacar en este punto es la consideración de la denominación neuroeducación para el espacio de intersección entre neurociencias y educación. Desde la perspectiva de la autora de este artículo no es aconsejable tomar como sinónimo neuroeducación y neurociencia educacional por varias razones. En primer lugar, neuroeducación es una denominación que en sí impresiona como centrada en lo neuro, en educar el cerebro, cuando sabemos muy bien los educadores que no educamos cerebros sino personas y que esas personas se encuentran en contexto, en situación, en interacción permanente con sus medios de vida. En segundo lugar, la denominación de neuroeducación tiene consecuencias sobre la práctica de esta transdisciplina, en tanto sugiere aplicaciones de lo neuro a lo educacional: parece llevar consigo una perspectiva aplicacionista de los estudios del cerebro sobre las aulas de clases. En tercer lugar, llamar neurociencia educacional a este ámbito de pensamiento y acción donde intersectan neurociencias y educación permite una amplitud de formas de concebirlo, tanto como una transdisciplina que produce conocimiento básico y traslacional como concibiéndolo como un enfoque o una aproximación a la praxis educacional. Esta amplitud de conceptualización, lejos de confundir, aporta una libertad de pensamiento interesante en situaciones de conformación del espacio, como ocurre actualmente, donde la restricción de miradas puede perjudicar el logro de objetivos.

Acordamos con una mirada cautelosa el establecimiento de relaciones neurociencias-educación, favoreciendo un punto de vista crítico de las neurociencias y de las ciencias en general (Schleim 2014).

Unos de los autores que desarrollan un punto de vista crítico de las neurociencias son Slaby y Gallagher (2015), quienes trabajan la mirada crítica asociándola con el concepto de mente socialmente extendida. Los autores señalan que el procesamiento cognitivo se extiende no solo por las tecnologías sino por la interacción intersubjetiva con otras mentes, dando lugar a lo que llaman mente socialmente extendida, especialmente cuando uno se enrola en o acopla con (coupling) instituciones (de donde sale el concepto de cognitive institutions, por ejemplo, la ciencia y el sistema legal, es decir, las reglas, prácticas y estructuras instituidas para propósitos cognitivos). La idea es que pensar la neurociencia 
Volumen 8, número 22, (223-245), septiembre-diciembre 2020 DOI: https://doi.org/10.22201/ceiich.24485705e.2020.22.76427 María de los Angeles Bacigalupe

como institución cognitiva puede colaborar con la construcción de una perspectiva crítica de la misma y de sus resultados.

Una mirada optimista sobre las relaciones entre neurociencia y educación es la que muestra Sousa $(2010,1)$ con las siguientes palabras: "The birth of educational neuroscience occurred with the help of the visionaries who have contributed to this book. And teaching will never be the same again."2

La fortaleza de esta última preposición entusiasma, pero es importante recordar la frase atribuida al pensador medieval Bernardo de Chartres: estamos subidos en los hombros de gigantes, suponiendo que desde allí podemos ver más lejos que ellos, pero de no haber estado estos gigantes no podríamos avanzar: no podemos despreciar lo hecho en siglos y milenios atrás por grandes educadores que fueron los que establecieron los mojones sobre los cuales hoy estamos en esta empresa de construcción de la neurociencia educacional.

Retomando lo comentado al comienzo de este artículo, los contextos actuales demandan competencias diferentes y es sobre la base de esa demanda y del avance del conocimiento que la enseñanza ya no será la misma.

\section{Estudios del comportamiento en la enseñanza de la neurociencia educacional}

Coincidiendo con Piaget (1978) y Lahitte (1989), Gomez-Marin, Paton, Kampff et al. (2014) señalan que el comportamiento es relacional en tanto implica estar en relación con el medioambiente y el aprovechamiento por parte del organismo de las affordances (cualidades de acción u oportunidades de comportarse) que ofrece el entorno. Claros ejemplos del concepto de affordances en relación con el comportamiento pueden verse en Chemero (2003), Mezuk, Abdou, Hudson et al. (2013), Montagne, Bastin y Jacobs (2008) y Oguro, Ward, Bracewel et al. (2009).

Además de relacional, el comportamiento es dinámico al ser un proceso que necesariamente involucra cambios, y esa dinámica se manifiesta en el espacio y el tiempo. Asimismo, el comportamiento es complejo y multidimensional, por lo cual, según Gomez-Marin et al. (2014), son decisivas en el estudio del comportamiento las técnicas de reducción dimensional.

Los mismos autores argumentan que si el cerebro es el que dirige el comportamiento, el comportamiento viene a constituirse en su principal función, por lo cual, si el problema de las neurociencias es comprender el funcionamiento cerebral, no alcanzará su objetivo quedándose en la explicación de los sistemas neurales: necesita realizar el vínculo con el comportamiento para lograr su meta. De este modo: "behavioral data is not simply a tool for helping neuros- 
cientists interpret brain data, but also the foundational problem of neuroscience" (Gomez-Marin et al. 2014, 1455).

Hay importantes aportes de la psicología a la neurociencia educacional que, si bien de base no están pensados con sostenimiento en los estudios del cerebro, contribuyen enormemente a la construcción de la disciplina. Estos son los estudios del reconocido psicólogo e intelectual ruso Lev S. Vygostky (1920-30/1993, 1931/1995, 1934/1995). Su concepto de zona de desarrollo próximo nos lleva a reflexionar sobre su existencia y realización en personas adultas, lo cual consideramos muy posible y de hecho lo hemos desarrollado en algunos de nuestros trabajos (Bacigalupe 2012; Bacigalupe, Lahitte y Tujague 2011).

Otros estudios sobre comportamiento que constituyen claves para la enseñanza de la neurociencia educacional son los estudios de Brenda Milner y Suzanne Corkin con H. M. (Cfr., por ejemplo, Corkin 1968 y 2013; Scoville y Milner 1957) y los trabajos sobre Phineas Gage (Cfr., por caso, Griggs 2015; Macmillan, 1999-2012). Así, también resultan realmente ilustrativos los casos descriptos por el maestro Alexander Luria (1979), los casos de Paul Broca (Cfr., por ejemplo, Dronkers, Plaisant, Iba-Zizen et al. 2007) y los de Roger Sperry y Michael Gazzaniga (Cfr., por caso, Gazzaniga 1998; Wolman 2012), entre muchos otros. También se hallan aproximaciones interesantes que sirven de referencia para la neurociencia educacional en los estudios de relación cerebro-comportamiento como por ejemplo los trabajos sobre cerebro, cerebelo y dislexia de Fawcet y Nicolson (2004) y Puente, Jiménez y Ardila (2009).

Los estudios con modelos animales no humanos son otros tantos ejemplos del estudio del comportamiento en neurociencia que pueden contribuir a la neurociencia educacional en función de problemáticas educacionales. Por ejemplo, Herrera, Miranda Contreras, Ramírez Martens et al. (2000), trabajando con ratas, mostraron que la desnutrición temprana afecta profundamente la morfología cerebelar a través de la alteración de los procesos de neurogénesis y sinaptogénesis. Atendiendo otra problemática nutricional y trabajando también con ratas, Vásquez, Sandoval, Smith et al. (2016) señalaron que el hecho de haber sufrido experiencias adversas en edades tempranas (alteración del vínculo madre-cría) o tardías (aislamiento) afectaba la fisiología del sistema de respuesta al estrés y la modulación de la conducta alimentaria, consumiendo alimentos hipercalóricos y apetecibles.

En nuestras investigaciones comportamentales hemos trabajado en el tema de la formación de memoria en personas con encefalopatía de Wernicke-Korsakoff, obteniendo claros indicios de las posibilidades de plasticidad que puede desplegar una persona con este tipo de alteración neurológica si se dan las condiciones contextuales adecuadas (Bacigalupe 2012; Bacigalupe, Tujague, Späth et al. 2013). 
Hoy en día, estamos trabajando en comportamiento con personas con enfermedad de Parkinson (Bacigalupe 2018; Bacigalupe, Dillon y Pujol 2010; Bacigalupe y Pujol 2014) desde el marco teórico neurológico de la kinesia paradojal (Cfr. Souques 1921; también otros autores como Asmus, Huber, Gasser et al. 2008; Ballanguer, Thobois, Baraduc et al. 2006; Bonanni, Thomas, Anzellotti et al. 2010; Bonanni, Thomas y Onofrj 2010; Schlesinger, Erikh y Yarnitsky 2007) y utilizamos los resultados de esos estudios para nuestra enseñanza.

Uno de los tópicos que en este momento estamos indagando es el comportamiento no verbal en personas con enfermedad de Parkinson (Cfr. antecedentes en Cleary, Poliakoff, Galpin et al. 2011; Humphries, Holler, Crawford et al. 2016), cuestión que tiene significación en espacios educacionales (Cfr. Goldin-Meadow 2014).

Dos de los grandes casos de la historia de las neurociencias: H. M., Tan (uno de los pacientes de Broca) y Phineas Gage, constituyen casos míticos (Jarrett 2015). Especialmente uno de ellos, el caso Gage ha dado lugar a la aparición de variadas versiones populares de la historia, lo que nos remite a nuestra sección anterior sobre neuromitos o, al menos, fábulas en la historia de la ciencia. Según la literatura, Phineas Gage era un capataz en la construcción de las vías del ferrocarril allá por mediados del siglo XIX, quien sufrió un terrible accidente cerca de Cavendish, Vermont, donde se dañó severamente parte de su lóbulo frontal. Gage vivió once años más luego del accidente y es esta etapa de su vida posterior al accidente la que ha dado lugar a su mítico personaje: se ha dicho que Gage llevó, luego del incidente, una vida errática y desorganizada, cuando, según estudios de Malcom Macmillan, uno de los investigadores que más se ha ocupado del tema, si bien Gage perdió su trabajo por sus cambios en la personalidad que ya no le permitían cumplir las funciones previamente asignadas, logró trabajos de responsabilidad donde mostró una conducta adaptada, muy lejos de lo que a veces se representa por el mítico Phineas Gage (Cfr. Macmillan 1999-2012; también, Grigss 2015; Jarret 2014).

Cuando trabajamos con casos como los mencionados, una de las cuestiones a destacar es que buscamos complementariedades de niveles de análisis y explicación más que privilegiar unos niveles por sobre otros o, lo que sería un gran error, despreciar unos niveles y subestimar otros. Resulta muy interesante, al respecto, el análisis de la complementariedad de las propuestas de Santiago Ramón y Cajal e Ivan Petrovich Pavlov que realizan Rozo y Rodríguez-Moreno (2015) desde la aproximación de la epistemología estratégica. Cabe destacar que los autores llaman epistemología estratégica al abordaje que propone trabajar las disciplinas científicas como estrategias para entender un problema más que como espacios aislados cada uno con un objeto de estudio diferente.

Estos autores señalan diálogos entre las perspectivas, pocas veces complementadas desde el análisis bibliográfico, de Cajal y de Pavlov. Mientras que Cajal 
mira la anatomía, Pavlov enfoca sobre la fisiología. Cajal estudia la célula neuronal e hipotetiza sobre la plasticidad nerviosa que subyace al comportamiento. Pavlov ve el problema de la conducta en la relación organismo-entorno aunque su postura, lejos de ser de co-construcción, supone que el entorno se impone al individuo y es el individuo el que cambia; sin embargo, esto no le impide (y hasta podría pensarse que favorece) su tarea de teorización sobre la plasticidad cerebral (término que quizás Pavlov no utilizaría en su momento pero que nosotros nos permitimos usarlo) que subyace a ese cambio. Cajal y Pavlov estudian desde diferentes puntos de vista la flexibilidad de la función nerviosa y conductual y ambos inspiran los trabajos de uno de los más grandes neurocientíficos que ha trabajado sobre aprendizaje y memoria de nuestra época: Eric Kandel (Rozo y Rodríguez-Moreno 2015).

De este modo, consideramos que la búsqueda de complementariedades y colaboraciones sirve mucho más a la construcción y la enseñanza de la neurociencia educacional que el señalamiento de diferencias e inconsistencias que, frecuentemente, son generadas a posteriori de las propuestas de los creadores de las grandes ideas.

Como al comienzo de este artículo señalábamos, nuestro objetivo es mostrar que la neurociencia educacional, si bien utiliza estudios del comportamiento humano mostrando un caso patológico a partir del cual se puede conocer mejor la función, no es una disciplina que se ocupe de las capacidades especiales y la discapacidad per se, aunque pueda colaborar en su abordaje educacional, claro está. En su artículo, Varma et al. (2008) ofrecen ejemplos de esta construcción que no se orienta a una educación especial necesariamente, ilustrando la neurociencia educacional en la práctica no solo en casos patológicos sino también hablando de las diferencias individuales en cerebros (y personas, obviamente) sanos.

De hecho, en nuestros estudios del comportamiento con personas con Parkinson, trabajamos desde la perspectiva de la salud, y no del déficit, dejando en claro nuestra orientación educacional, prospectiva, que mira hacia adelante, destacando el potencial de aprendizaje más que la dificultad. Ahora bien, somos conscientes de que solo es posible este abordaje educacional partiendo desde un conocimiento fundado de la neurociencia, pues solo conociendo somos capaces de pensar en toda su plenitud la posibilidad de la que la biología dota al individuo en su relación con el medio. Estamos absolutamente convencidos de que la biología es posibilidad. Lejos de poner límites al desarrollo, los datos de las neurociencias aportan conocimientos que permiten ampliar el universo de potencialidades del organismo relacionado con su entorno, cualidad que llamamos plasticidad. Como responsables de la educación de las personas, es nuestro métier el descubrimiento de las potencialidades de las mismas a partir de sus diferencias individuales, trátese de personas neurológicamente sanas (en el 
sentido en el que toma la medicina el concepto de salud) o no. Como bien nos han enseñado maestros de la literatura y la neurociencia como Oliver Sacks (Cfr. por ejemplo, Sacks 2007, 2008 y 2011) y también Suzanne Corkin (2013) y otros, el objetivo es ir a la persona en sí con toda su humanidad y sus contextos más que al déficit o el caso raro u anecdótico.

De este modo, el abordaje de los estudios del comportamiento humano para la construcción de la neurociencia educacional no es el mismo que para la medicina o la psicología: requiere de un trabajo previo y un estudio profundo de los casos estudiados, a fin de presentarlos para aprender de ellos las posibilidades que la biología brinda, o, mejor dicho, los distintos modos de co-construcción organismo-entorno, generando un conocimiento fundado y una concepción de humanidad que es lo que formará parte del background del educador al momento de enfrentar una situación educativa concreta y tener que tomar decisiones.

\section{Conclusión}

En un artículo previo, proponíamos el concepto neurociencia de y con la educación (Bacigalupe y Mancini 2014), para diferenciarlo de la neurociencia de la educación y otras formas de llamar a este campo que le dan connotaciones aplicacionistas diferentes a la tan buscada transdisciplinariedad. Actualmente, la autora de este artículo considera que los avances sobre la neurociencia educacional permiten adoptar ese nombre para el campo en construcción donde no prevalece una disciplina sobre otra sino que se genera conocimiento nuevo con una identidad educacional, propia y original. Consideramos que otorgar entidad a este campo del saber permitirá que sea reconocido por otras ciencias de la educación y disciplinas auxiliares y colaboradoras con las cuales trabajará en conjunto para la formación de los educadores y la toma de decisiones educacionales.

Los estudios del comportamiento humano constituyen elementos ineludibles para la enseñanza de la neurociencia educacional. Nuestro desafío en la construcción de la transdisciplina es que los casos que trabajemos no sean tomados como ejemplos de intervención pedagógica per se sino como lo que son: ilustraciones de cómo funciona y no funciona el cerebro en ciertos contextos y bajo determinadas circunstancias y demostraciones de la plasticidad cerebral y las posibilidades de las personas de reconstruir sus relaciones con el entorno en función del despliegue de sus potencialidades humanas. No negamos que la neurociencia educacional aporte elementos para la mirada de la educación con personas con discapacidad o con capacidades especiales pero eso no significa que se identifique con ese área de la educación.

Todos los sujetos de la educación (y no solo las personas con capacidades especiales y discapacidades) tenemos biología y necesitamos comportarnos en 
relación con un entorno que nos constituye y que a la vez contribuimos a construir, en una relación dialógica de co-construcción.

La neurociencia educacional nos da elementos para pensar la educación de las personas y los comportamientos humanos que contribuyen a las relaciones de aprendizaje en los distintos escenarios pedagógicos, tanto formales como no formales e informales y a lo largo y a lo ancho de la curva normal. Conocer la biología humana es conocer las potencialidades de las personas en sus contextos, pues estamos absolutamente convencidos de que la biología es posibilidad. Tradicionalmente se ha dicho lo contrario: la biología representa el límite a la potencialidad humana. Nuestra postura es totalmente la contraria: la biología que las neurociencias nos aportan representa la posibilidad del despliegue de las potencialidades de los individuos, demuestra que los cerebros humanos son por naturaleza cerebros sociales y que las disociaciones entre naturaleza y cultura, biología y sociedad y otras por el estilo son tan construcciones humanas como la existencia de las brujas en su momento. Los avances de las ciencias permitieron derribar verdades asumidas (mitos) en su momento y eso, suponemos, se repite permanentemente a lo largo de la historia. Obviamente, siendo coherentes con nuestras posiciones ya expresadas, esa construcción es transdisciplinaria y por ende no esperamos que haya un dominio de un conocimiento por encima de otros (las neurociencias por sobre la educación), así como tampoco que podamos echar las culpas a las neurociencias de los neuromitos que generamos desde la educación. Suponemos que la construcción de la neurociencia educacional como espacio y aproximación legítima, original y transdisciplinaria permitirá que estos dos errores mencionados anteriormente no volvamos a cometerlos.

De este modo, el enfoque de la enseñanza no será el mismo, pero no será el mismo no porque hasta ahora hayamos estado equivocados y vengan las neurociencias a demostrarnos sus saberes y a corregirnos, sino porque desde la misma neurociencia educacional generamos un conocimiento nuevo que nos permite, en inter-diálogo con otras ciencias de la educación, y frente a la demanda de los contextos actuales, dar una nueva mirada al quehacer educacional. Por ejemplo, tradicionalmente hemos enseñado la metodología del estudio como una disciplina instrumental donde los contenidos principales están basados en las técnicas de estudio, algo tan necesario porque vemos que al llegar a los estudios superiores nuestros alumnos no cuentan con estrategias de aprendizaje para adquirir grandes cúmulos de conocimiento teórico como exige la universidad. Sin embargo, un enfoque novedoso de la metodología del estudio podría ser comenzar, en vez de con la enseñanza de las técnicas, con el análisis del concepto de motivación (Cfr. Kim 2013) y estrategias de conocimiento de sí mismo, no para fomentar un solipsismo y un encierro en la idea del que uno puede todo, lo cual sería un gran error, sino para justamente lo contrario: conocerse a sí mismo para potenciar las propias 
Volumen 8, número 22, (223-245), septiembre-diciembre 2020 DOI: https://doi.org/10.22201/ceiich.24485705e.2020.22.76427 María de los Angeles Bacigalupe

capacidades y apoyarse en el otro en el trabajo en equipo, estrategia de colaboración tan necesaria de desarrollar en los contextos profesionales y científicos actuales y para lo cual no solemos preparar a nuestros alumnos. Como al comienzo de nuestro trabajo señalábamos, con este enfoque apoyamos una aproximación metacognitiva de la enseñanza y la ponemos en evidencia en este ejemplo que acabamos de mencionar. Conocerse a sí mismo, conocer las propias posibilidades, las potencialidades, los modos de percibir, de memorizar, de aprender, de responder, de sentir, de entender al otro, analizar las motivaciones y cómo uno se aproxima al mundo que lo rodea, confiamos en que ayudará a reconstruir las relaciones con el entorno, desarrollar un pensamiento crítico hacia sí y en relación con el contexto y comprender, respetar y favorecer la diversidad y su riqueza.

\section{Referencias}

Aristóteles. 1970/s. «IV aC. Libro VI.» En Ética a Nicómaco, edición bilingüe y traducción por M. Araujo y J. Marías, introducción y notas de J. Marías. Madrid: Instituto de Estudios Políticos, 89-101.

Asmus, F., H. Huber, T. Gasser et al. 2008. «Kick and rush: Paradoxical kinesia in Parkinson disease.» Neurology, 71(9): 695. https://doi.org/10.1212/01.wnl.0000324618.88710.30

Bacigalupe, M. A. 2001/2. "How neuroscientific studies on memory can help teaching.» IATEFL Issues, 164: 12.

Bacigalupe, M. A. 2018. "Comportamiento no verbal en personas con enfermedad de Parkinson: desafío y posibilidad de comunicación.» Duazary: Revista Internacional de Ciencias de la Salud, 15(2): 195-209.

https://doi.org/10.21676/2389783X.2104.

Bacigalupe, M. A. y S. Pujol. 2014. «El movimiento desde la perspectiva de la neurociencia social cognitiva: el caso de la enfermedad de Parkinson.» Vertex-Revista Argentina de Psiquiatría, 25(118): 429-436.

Bacigalupe, M. A. y V. Mancini. 2014. «Contribuciones para la construcción de un enfoque de las neurociencias de y con la educación en la formación universitaria de pregrado en ciencias de la educación.» Profesorado: Revista de curriculum y formación del profesorado, 18(1): 431-440.

Bacigalupe, M. A., J. L. Dillon y S. Pujol. 2010. «Learning perspective on paradoxical kinesia (PK) in Parkinson's disease (PD).» Movement Disorders, 25(supl. 2): S352-S353.

Bacigalupe, M. A., H. B. Lahitte y M. P. Tujague. 2011. «Enfoque de la evaluación dinámica y sus raíces interaccionistas como perspectiva metodológica en la investigación y práctica educacionales.» Revista de Educación y Desarrollo, 16: 27-33. 
Bacigalupe, M. A., M. P. Tujague, G. M. Späth et al. 2013. «Behavioural research on human working memory: Mixing qualitative and quantitative methods.» Liberabit: Lima (Perú), 19(2): 195-203.

Ballanguer, B., S. Thobois, P. Baraduc et al. 2006. «Paradoxical kinesis' is not a hallmark of Parkinson's disease but a general property of the motor system.» Movement Disorders, 21(9): 1490-1495.

Barsalow, L. W. (2010). «Grounded cognition: Past, present and future.» Topics in Cognitive Science, 2: 716-24. https://doi.org/10.1111/j.1756-8765.2010.01115.x

Bonanni, L., A. Thomas, F. Anzellotti et al. 2010. «Protracted benefit from paradoxical kinesia in typical and atypical parkinsonisms.» Neurological Sciences, 31(6): 751-756. https://doi.org/10.1007/s10072-010-0403-5

Bonanni, L., A. Thomas y M. Onofrj. 2010. «Paradoxical kinesia in parkinsonian patients surviving earthquake.» Movement Disorders, 25(9): 1302-1304. https://doi.org/10.1002/mds.23075

Bransford, J. D., A. L. Brown y R. R. Cocking, R. R. (eds.). 2000. How people learn: brain, mind, experience, and school: expanded edition. Washington, D.C.: National Academy Press.

Burgos, J. A. 2015. «Greasing the skids of the musical mind: connecting music learning to mind brain education.» General Music Today, 29(1): 5-11. https://doi.org/10.1177/1048371314566236

Butterworth, B. y A. Tolmie. 2014. «Introduction.» En D. Mareschal, B. Butterworth y A. Tolmie (eds.), Educational neuroscience (E-book, versión Kindle). UK: Wiley Blackwell, loc. 358-595.

Caine, R. N. y G. Caine. 1994. "Principles of brain based learning.» En Making connections: Teaching and the human brain. EUA: Innovative Learning Publications, 87-96.

Carreón Mendoza, H. y R. Melgoza Ramos. 2012. «México hacia una sociedad del conocimiento.» Nóesis: Revista de Ciencias Sociales y Humanidades, 21(41): 120135. http://erevistas.uacj.mx/ojs/index.php/noesis/article/view/209/200

Chemero, A. 2003. "An outline of a theory of affordances.» Ecological Psychology, 15(2): 181-195.

Cleary, R. A., E. Poliakoff, A. Galpin et al. 2011. «An investigation of co-speech gesture production during action description in Parkinson's disease.»Parkinsonism \& Related Disorders, 17(10): 753-756.

https://doi.org/10.1016/j.parkreldis.2011.08.001

Corkin, S. 1968. «Acquisition of motor skill after bilateral medial temporal-lobe excision.» Neuropsychologia, 6, pp. 255-65.

Corkin, S. 2013. Permanent present tense: The man with no memory, and what he taught the world (E-book, versión Kindle). UK: Penguin.

Damasio, A. 2008. «La hipótesis del marcador somático.» En El error de Des- 
cartes: La emoción, la razón y el cerebro humano. Barcelona: Crítica, 196-235

De la Fuente Arias, J., M. M. Vera Martínez y M. Cardelle-Elawar. 2012. «Aportaciones de la psicología de la innovación y del emprendimiento a la educación, en la sociedad del conocimiento.» Electronic Journal of Research in Educational Psychology, 10(3): 941-966.

Dekker, S., N. Lee, P. Howard-Jones et al. 2012. "Neuromyths in education: Prevalence and predictors of misconceptions among teachers.» Frontiers in Psychology, 3(art. 428): 1-8. https://doi.org/10.3389/fpsyg.2012.00429

Dommett, E. J., I. M. Devonshire, C. R. Plateau et al. 2011 . «From scientific theory to classroom practice.» The Neuroscientist, 17(4): 382-388. https://doi.org/10.1177/1073858409356111

Dronkers, N. F., O. Plaisant, M. T. Iba-Zizen et al. 2007. «Paul Broca's historic cases: high resolution MR imaging of the brains of Leborgne and Lelong.» Brain, 130: 1432-1441. https://doi.org/10.1093/brain/awm042

Fawcet, A. y R. Nicolson, R. 2004. «El cerebelo: su implicación en la dislexia.» Revista Electrónica de Investigación Psicoeducativa y Psicopedagógica, 2(2): 35-58.

Fischer, K. W. y K. Heikkinen. 2010. «The future of educational neuroscience.» En D. A. Sousa (ed.), Mind, brain \& education: Neuroscience implications for the classroom. Bloomington, IN, EUA: Solution Tree Press, 248-270

Garay, C., Bacigalupe, M. A., R. Istvan et al. 2015. «Sobre la integración entre ciencias naturales y sociales.» En A. Pereyra. Gonnet (ed.), Prácticas pedagógicas y políticas educativas. Investigaciones en el territorio bonaerense. Argentina: UNIPE Editorial Universitaria, 463-496. http://editorial.unipe.edu.ar/wp-content/uploads/2015/03/Pr\%C3\%Al cticas-pedag\%C3\%B3gicas-y-pol\%C3\%ADticas-educativas.-Investigaciones-en-el-territorio-bonaerense-UNIPE-2015.pdf

Gazzaniga, M. S. 1998. «The split brain revisited.» Scientific American, 279(1): 50-55.

Goldin-Meadow, S. 2014. «How gesture works to change our minds.» Trends in Neuroscience and education, 3: 4-6. https://doi.org/10.1016/j.tine.2014.01.002

Gomez-Marin, A., J. J. Paton, A. R. Kampff et al. 2014. «Big behavioral data: psychology, ethology and the foundations of neuroscience.» Nature Neuroscience, 17(11): 1455-1462. https://doi.org/10.1038/nn.3812

Griggs, R. A. 2015. «Coverage of the Phineas Gage story in introductory psychology textbooks: was Gage no longer Gage?» Teaching of Psychology, 42(3): 195-202. https://doi.org/10.1177/0098628315587614

Herrera, F. A., L. Miranda Contreras, L. Ramírez Martens et al. 2000. «Cambios bioquímicos y morfológicos en el sistema nervioso central de ratas desnutridas durante la gestación y lactancia.» Interciencia, 25(2): 85-91. http://www.redalyc.org/articulo.oa?id=33904406 
Humphries, S., J. Holler, T. J. Crawford et al. 2016. «A third-person perspective on co-speech action gestures in Parkinson's disease.» Cortex, 78: 44-56. https://doi.org/10.1016/j.cortex.2016.02.009

Hruby, G. 2012. «Three requirements for justifying an educational neuroscience.» British Journal od Educational Psychology, 82: 1-23. https://doi.org/10.1111/j.2044-8279.2012.02068.x

Jarrett, C. 2015. Great Myths of the Brain. UK: Wiley Blackwell.

Kalbfleisch, L. 2015. «Editorial note for the research topic special issue on educational neuroscience, constructivism, learning, and the mediation of learning and creativity in the 21 st century.» Frontiers in Psychology, 6 (art. 133): 1-2. https://doi.org/10.3389/fpsyg.2015.00133

Kim, S. 2013. «Neuroscientific model of motivational process.» Frontiers in Psychology (eCollection), 4(art. 98): 1-12. https://doi.org/10.3389/fpsyg.2013.00098

Knox, R. 2016. "Mind, brain, and education: A transdisciplinary field.» Mind, Brain, and Education, 10(1): 4-9. https://doi.org/10.1111/mbe.12102

Lahitte, H. B., J. A. Hurrell y A. Malpartida. 1989. Relaciones 2: Crítica y expansión de la ecología de las ideas. Argentina: Ediciones Nuevo Siglo.

Lewontin, R. 2000. Genes, organismo y ambiente: Las relaciones de causa y efecto en biología. Barcelona: Gedisa.

Luria, A. R. 1979. El cerebro humano y los procesos psíquicos: Análisis neuropsicológico de la actividad consciente. Barcelona: Fontanella S. A.

Macmillan, M. 1999-2012. The Phineas Gage information page. The University of Akron, Ohio Polytechnic University. https://www.uakron.edu/gage/ (19 de agosto, 2015).

Meyer, F. 1977. «El concepto de adaptación.» En Piaget, J., P. A. Osterrieth, J. Nuttin et al. (eds.), Los procesos de adaptación. Buenos Aires: Nueva Visión, 13-27

Mezuk, B., C. M. Abdou, D. Hudson et al. 2013. "White box” epidemiology and the social neuroscience of health behaviors: The environmental affordances model.» Society and Mental Health, 3(2): 79-95.

https://doi.org/10.1177/2156869313480892

Montagne, G., J. Bastin, Y. D. M. Jacobs. 2008. «What is visual anticipation and how much does it rely on the dorsal stream?» International Journal of Sport Psychology, 39(2): 149-156. http://www.ism.univmed.fr/IMG/pdf/Montagne Bastin_Jacobs_IJSP_2008.pdf

Oguro, H., R. Ward, M. Bracewel et al. 2009. «Automatic activation of motor programs by object affordances in patients with Parkinson's disease.» Neuroscience Letters, 463(1-10): 35-36.

Piaget, J. 1978. Behavior and evolution. Nueva York: Panteon Books.

Pincham, H. L., A. A. Matejko, A. Obersteiner et al. 2014. «Forging a new path for educational neuroscience: An international young researcher perspective 
on combining neuroscience and educational practices.» Trends in Neuroscience and Education, 3(1): 28-31. https://doi.org/10.1016/j.tine.2014.02.002 Puente, A., V. Jiménez y A. Ardila. 2009. «Anormalidades cerebrales en sujetos disléxicos.» Revista Latinoamericana de Psicología, 41(1): 27-45.

Rodgers, D. L. 2015. «The biological basis of learning: Neuroeducation through simulation.» Simulation \& Gaming, 46(2): 175-186.

https://doi.org/10.1177/1046878115590585

Rodríguez-Ponce, E. 2009. «El rol de las universidades en la sociedad del conocimiento y en la era de la globalización: Evidencia desde Chile.»Interciencia, 34(11): 822-829.

Rozo, J. A. y A. Rodríguez-Moreno. 2015. «Santiago Ramón y Cajal e Ivan Petrovich Pavlov: ¿existe complementariedad entre sus teorías?» Revista de Neurología, 61: 125-36.

Sacks, O. 2007. The man who mistook his wife for a hat, reimpr. Kent, UK: Picador.

Sacks, O. 2008. Musicophilia: Tales of music and the brain, edición rev. y aum. Nueva York: Vintage Books.

Sacks, O. 2011 . An anthropologist on Mars: Seven paradoxical tales (E-book, version Kindle). Londres: Picador.

Schleim, S. 2014. «Critical neuroscience - or critical science? A perspective on the perceived normative significance of neuroscience.» Frontiers in Human Neuroscience, 8: 1-6. https://doi.org/10.3389/fnhum.2014.00336

Schlesinger, H., I. Erikh y D. Yarnitsky. 2007. «Paradoxical kinesia at war.» Movement Disorders, 22(16): 2394-2397.

Scoville, W. B. y B. Milner. 1957. «Loss of recent memory after bilateral hippocampal lesions.» Journal of Neurology, Neurosurgery and Psychiatry, 20(11): 11-21.

Slaby, J. y S. Gallagher. 2015. «Critical neuroscience and socially extended minds.» Theory, Culture \& Society, 32(1): 33-59.

https://doi.org/10.1177/0263276414551996.

Souques, A. A. 1921. «Rapport sur les syndromes parkinsoniens.»Revue Neurologique, 37: 534-573.

Sousa, D. A. 2010. «Introduction.» En D. A. Sousa (ed.), Mind, brain \& education: Neuroscience implications for the classroom. Bloomington, IN, USA: Solution Tree Press, 1-7.

Spitzer, M. 2012. «Editorial: Education and neuroscience.» Trends in Neuroscience and Education, 1(1): 1-2. https://doi.org/10.1016/j.tine.2012.09.002

Tokuhama-Espinosa, T. 2011 . "Why mind, brain and education science is the "new" brain-based education.» New Horizons for Learning, 9(1): 1-11. http://education.jhu.edu/PD/newhorizons/Journals/Winter2011/Tokuhama1 
Turner, J. S. 2000. The extended organism: The physiology of animal-built structures. USA: Harvard University Press.

Varma, S., B. D. McCandliss y D. L. Schwartz. 2008. «Scientific and pragmatic challenges for bridging education and neuroscience.» Educational Researcher, 37(3): 140-152. https://doi.org/10.3102/0013189X08317687

Vásquez, B., C. Sandoval, R. L. Smith et al. 2016. «Efectos de las experiencias adversas temprana y tardía en la conducta de ingesta de ratas sometidas a estrés durante su adultez.»Interciencia, 41(3): 177-183. http://www.redalyc. org/articulo.oa?id=33944256006

Vygostky, L. S. 1934/1995. Pensamiento y lenguaje. Barcelona: Paidós.

Vygostky, L. S. 1920-30/1993. "The fundamentals of defectology.» En R. W. Rieber (ed.), The collected works of L. S. Vygotsky Volume 2. Nueva York: Plenum Press, 27-291.

Vygostky, L. S. 1931/1995. Historia del desarrollo de las funciones psíquicas superiores (Obras escogidas, III). Madrid: Visor.

Wolman, D. 2012. «A tale of two halves.» Nature, 483: 260-263. 